\title{
A NOTE ON CONGRUENCES ON ORTHODOX SEMIGROUPS
}

\author{
by D. B. McALISTER
}

(Received 24 August, 1983)

C. Eberhart and W. Williams [3] showed that the least inverse semigroup congruence $\mathscr{Y}$, on an orthodox semigroup $S$, plays a very important role in determining the structure of the lattice of congruences on $S$. In this note we show that their results can be applied to give an explicit construction for the idempotent separating congruences on $S$ in terms of idempotent separating congruences on $S / \mathscr{Y}$.

The description which we obtain for idempotent separating congruences on orthodox semigroups is used to give an alternative characterization, for idempotent separating congruences on the semidirect product of a band by a group, to that given by $R$. McFadden [7].

The work was partially carried out while the author was visiting the University of Glasgow in May 1983. He is grateful for the hospitality he received there.

1. Preliminary results. We shall assume familiarity with the basic theory of orthodox and regular semigroups as found, for example, in the books by Clifford and Preston [2] and Howie [5]. Thus, in particular, we shall denote by $V(a)$ the set of inverses of an element $a$ in a regular semigroup $S$.

T. E. Hall [4] has shown that the minimum inverse semigroup congruence on an orthodox semigroup $S$ is given by:

$$
(a, b) \in \mathcal{Y} \text { if and only if } V(a)=V(b) .
$$

He has shown further that a regular semigroup $S$ is orthodox if and only if $V(a) \cap$ $V(b) \neq \varnothing$ implies $V(a)=V(b)$. Thus, when $S$ is orthodox, $a$ and $b$ in $S$ are $y$-related if and only if $V(a) \cap V(b) \neq \varnothing$. In addition, Reilly and Scheiblich [10] have shown that a regular semigroup $S$ is orthodox if and only if each inverse of an idempotent is itself idempotent.

A congruence $\rho$, on a regular semigroup $S$, is idempotent separating if no two distinct idempotents of $S$ belong to the same $\rho$-class. W. D. Munn [9] has shown that a congruence $\rho$ on $S$ is idempotent separating if and only if it is contained in Green's relation $\mathscr{H}$. Thus there is a greatest idempotent separating congruence $\mu$ on $S$. Munn shows that the idempotent separating congruences on a regular semigroup commute. This latter result has been generalized by Eberhart and Williams to include certain other congruences on orthodox semigroups.

RESULT 1.1 [3]. Let $\rho$ and $\sigma$ be congruences on an orthodox semigroup $S$ and suppose that $\rho \subseteq \mathscr{Y}$ and $\sigma \subseteq \mu$. Then $\rho^{\circ} \sigma=\sigma \circ \rho$ so that the join $\rho \vee \sigma$ of $\rho$ and $\sigma$, in the lattice of congruences on $S$, is given by $\rho \vee \sigma=\rho \circ \sigma$. In particular, $\mathscr{Y} \vee \mu=\mathscr{Y} \circ \mu=\mu \circ \mathcal{O}$.

The main thrust of [3] is to show that congruences on an orthodox semigroup $S$ are

Glasgow Math. J. 26 (1985) 25-30. 
determined by their interaction with $\mu$ and $\mathscr{y}$. For use later in this paper, we shall require one of their separation results.

RESULT 1.2 [3]. Let $\sigma$ and $\tau$ be idempotent separating congruences on an orthodox semigroup $S$ and suppose that $\sigma \vee \mathcal{Y}=\tau \vee \mathscr{Y}$. Then $\sigma=\tau$.

J. Meakin has described the minimum idempotent separating congruence on an orthodox semigroup, as follows.

ReSUlt 1.3 [8]. Let $S$ be an orthodox semigroup and let $a, b \in S$. Then $(a, b) \in \mu$ if and only if there exist $a^{\prime} \in V(a), b^{\prime} \in V(b)$ such that $a^{\prime} e a=b^{\prime} e b$ for each idempotent $e$ in $S$.

2. Idempotent separating congruences. Suppose that $a$ and $b$ are $\mathscr{H}$-equivalent elements in any semigroup $S$ and that they have a common inverse $x$. Then one easily calculates that

$$
\begin{aligned}
a=a \times a=a \times b x a & =b \times a & & \text { since } a \mathscr{R} b \text { implies } b=a x b \\
& =b & & \text { since } a \mathscr{L} b \text { implies } b=b x a .
\end{aligned}
$$

Hence, on an orthodox semigroup, $\mathscr{H} \cap \mathcal{Y}$ is the identical congruence. We shall use this observation, and the results in Section 1, to describe the idempotent separating congruences on an orthodox semigroup $S$ in terms of idempotent separating congruences on $S / 9$.

Another consequence of this remark is given in Lemma 2.1. We shall find it useful later.

Lemma 2.1. Let $a, b$ be $\mathscr{H}$-equivalent elements of $a$ semigroup $S$ and let $\rho$ be $a$ congruence on $S$. Suppose that there are inverses $a^{\prime}$ of $a$ and $b^{\prime}$ of $b$, respectively, such that $\left(a^{\prime}, b^{\prime}\right) \in \rho$. Then $(a, b) \in \rho$.

Proof. In $S / \rho$, the elements $a \rho$ and $b \rho$ are $\mathscr{H}$-equivalent and have a common inverse $a^{\prime} \rho=b^{\prime} \rho$. Thus $a \rho=b \rho$.

In the remainder of this paper, $S$ will denote an orthodox semigroup and $T$ will denote its maximum inverse semigroup homomorphic image: that is, $T=S / \mathscr{Y}$.

For any congruence $\sigma$ on $T$, we obtain a congruence $\bar{\sigma}$ on $S$ according to the prescription

$$
(a, b) \in \overline{\boldsymbol{\sigma}} \quad \text { if and only if }(a \mathscr{Y}, b \mathcal{Y}) \in \sigma .
$$

We shall denote by $\mu^{*}$ the congruence $(\mu \vee \mathscr{Y}) / \mathscr{Y}$ on $T$. Thus

$$
(a \mathscr{Y}, b \mathcal{Y}) \in \mu^{*} \quad \text { if and only if } \quad(a, b) \in \mu \vee \mathscr{Y} \text {. }
$$

LEMMA 2.2. $\mu^{*}$ is an idempotent separating congruence on $T$.

Proof. Let $A, B$ be idempotents of $T$. Then, by Lallement's lemma there exist idempotents $e \in A, f \in B$. Thus $(A, B) \in \mu^{*}$ implies $(e, f) \in \mu \vee \mathscr{Y}=\mu \circ \mathscr{Y}$. Thus there exists $c \in S$ such that $(e, c) \in \mu,(c, f) \in \mathscr{Y}$. But, since $S$ is orthodox, $(c, f) \in \mathscr{Y}$ implies $c$ is idempotent. Then, since no $\mathscr{H}$-class contains more than one idempotent, $(e, c) \in \mu$ implies $c=e$. Hence $(e, f) \in \mathscr{Y}$ which gives $A=B$. 
LEMMA 2.3. Let $a, b \in S$. Then $\left(a \mathscr{Y}, b^{\mathscr{Y}}\right) \in \mu^{*}$ if and only if there exist $a^{\prime} \in V(a)$, $b^{\prime} \in V(b)$ such that $\left(a^{\prime}, b^{\prime}\right) \in \mu$.

Proof. Suppose that $(a \mathscr{Y}, b \mathscr{Y}) \in \mu^{*}$. Then, by Result $1.1,(a, b) \in \mu \circ \mathscr{Y}$. Thus there exists $c \in S$ such that $(a, c) \in \mu,(c, b) \in \mathscr{Y}$. Since $(a, c) \in \mu$, there exist inverses $a^{\prime}$ of $a$ and $c^{\prime}$ of $c$ such that, for all idempotents $e \in S, a^{\prime} e a=c^{\prime} e c$ (by Result 1.3). But this means $\left(a^{\prime}, c^{\prime}\right) \in \mu$. On the other hand, since $(c, b) \in \mathscr{Y}, V(c)=V(b)$ so that $c^{\prime} \in V(b)$. Hence there exists $b^{\prime}\left(=c^{\prime}\right)$ in $V(b)$ such that $\left(a^{\prime}, b^{\prime}\right) \in \mu$.

Conversely, suppose that there exist $a^{\prime} \in V(a), b^{\prime} \in V(b)$ such that $\left(a^{\prime}, b^{\prime}\right) \in \mu$. Let $c=b a^{\prime} a$. Then, since $a^{\prime} a \mathscr{R} a^{\prime} \mu b^{\prime} \mathscr{R} b^{\prime} b$, it follows from Green's Lemma that $a \mathscr{L} c \mathscr{R} b$. Now, let $d=a a^{\prime} c$; then, since $a a^{\prime} \mathscr{L} a^{\prime} \mu b^{\prime} \mathscr{L} b b^{\prime}$, it follows, again from Green's Lemma, that $a \mathscr{H} d$.

Further $b^{\prime} \in V(d)$ for

$$
d b^{\prime} d=a a^{\prime} b a^{\prime} a b^{\prime} a a^{\prime} b a^{\prime} a=a a^{\prime} b b^{\prime} b a^{\prime} a=d,
$$

since $a^{\prime} a \mathscr{R} b^{\prime} b$ implies $a^{\prime} a b^{\prime}=b^{\prime}$ and $a a^{\prime} \mathscr{L} b b^{\prime}$ implies $b^{\prime} a a^{\prime}=b^{\prime}$, and similarly $b^{\prime}=b^{\prime} d b^{\prime}$. Hence $a$ and $d$ are $\mathscr{H}$-equivalent elements of $S$, with $\mu$-equivalent inverses. Thus, by Lemma $2.1,(a, d) \in \mu$ and so, since $(d, b) \in \mathscr{Y},(a, b) \in \mu \circ \mathscr{Y}$.

THEOREM 2.4. Let $S$ be an orthodox semigroup and let $\tau$ be an idempotent separating congruence on $T=S / \mathcal{Y}$, such that $\tau \subseteq \mu^{*}$. Then $\mathscr{H} \cap \bar{\tau}$ is an idempotent separating congruence on $S$.

Conversely, if $\sigma$ is an idempotent separating congruence on $S$, there is a unique congruence $\tau$ on $T$, contained in $\mu^{*}$, such that $\sigma=\mathscr{H} \cap \bar{\tau}$.

Proof. Set $\tau^{\#}=\mu \cap \bar{\tau}$. Then, clearly, $\tau^{\#}$ is a congruence on $S$ and is contained in $\mathscr{H} \cap \bar{\tau}$. Conversely, suppose that $(a, b) \in \mathscr{H} \cap \bar{\tau}$. Then $(a, b) \in \mathscr{H}$ and, since $\tau \subseteq \mu^{*}$, $(a \mathscr{Y}, b \mathscr{Y}) \in \mu^{*}$. Thus, by Lemma 2.3, there exist $a^{\prime} \in V(a), b^{\prime} \in V(b)$ such that $\left(a^{\prime}, b^{\prime}\right) \in \mu$. Hence $a$ and $b$ are $\mathscr{H}$-equivalent elements with $\mu$-equivalent inverses and so, by Lemma $2.1,(a, b) \in \mu$. It follows therefore that $\mathscr{H} \cap \bar{\tau} \subseteq \mu \cap \bar{\tau}$ so that, in fact, $\mathscr{H} \cap \bar{\tau}=\mu \cap \bar{\tau}$ is a congruence on $S$. Since it is contained in $\mu$, it is evidently idempotent separating.

Now suppose that $\sigma$ is an idempotent separating congruence on $S$. Then, since $\sigma \subseteq \mu$, $\tau=(\sigma \vee \mathcal{Y}) / \mathscr{Y} \subseteq(\mu \vee \mathscr{Y}) / \mathscr{Y}=\mu^{*}$. Further

$$
(\bar{\tau} \cap \mathscr{H}) \vee \mathscr{Y}=(\bar{\tau} \cap \mu) \vee \mathscr{Y}=[(\sigma \vee \mathscr{Y}) \cap \mu] \vee \mathscr{Y} .
$$

But $(a, b) \in(\sigma \vee \mathscr{Y}) \cap \mu$ implies $(a, c) \in \sigma,(c, b) \in \mathscr{Y}$, for some $c \in S$, and $a \mathscr{H} b$. Since $(a, c) \in \sigma$ implies $a \mathscr{H} c, b$ and $c$ are thus $\mathscr{H}$-equivalent elements with an inverse in common. Hence $b=c$ so that $(a, b) \in \sigma$. It follows that $(\bar{\tau} \cap \mathscr{H}) \vee \mathscr{Y} \subseteq \sigma \vee \mathscr{Y}$. On the other hand, $\sigma \subseteq(\sigma \vee \mathscr{Y}) \cap \mu$ so that $\sigma \vee \mathscr{Y} \subseteq[(\sigma \vee \mathscr{Y}) \cap \mu] \vee \mathscr{Y}=(\bar{\tau} \cap \mathscr{H}) \vee \mathscr{Y}$. Hence $\sigma \vee \mathscr{Y}=$ $(\bar{\tau} \cap \mathscr{H}) \vee \mathscr{Y}$ and so, by Result $1.2, \sigma=\bar{\tau} \cap \mathscr{H}$.

Finally, suppose that $\bar{\rho} \cap \mathscr{H} \subseteq \bar{\tau} \cap \mathscr{H}$ where $\rho, \tau$ are congruences on $T$, contained in $\mu^{*}$. Then $(a \mathscr{Y}, b \mathscr{Y}) \in \rho$ implies $(a \mathscr{Y}, b \mathscr{Y}) \in \mu^{*}$ so that, by Lemma 2.3 , there exist $a^{\prime} \in V(a)$, $b^{\prime} \in V(b)$ such that $\left(a^{\prime}, b^{\prime}\right) \in \mu$. But, since $\rho$ is a congruence on an inverse semigroup, $(a \mathscr{Y}, b \mathcal{Y}) \in \rho$ implies $\left(a^{\prime} \mathscr{Y}, b^{\prime} \mathscr{Y}\right) \in \rho$. Hence $\left(a^{\prime}, b^{\prime}\right) \in \mu \cap \bar{\rho} \subseteq \mu \cap \bar{\tau}$, since $\mu \cap \bar{\rho}=\mathscr{H} \cap \bar{\rho} \subseteq$ $\mathscr{H} \cap \bar{\tau}=\mu \cap \bar{\tau}$. This in turn implies $\left(a^{\prime} \mathscr{Y}, b^{\prime} \mathscr{Y}\right) \in \tau$, whence $\left(a \mathscr{Y}, b^{\mathscr{Y}}\right) \in \tau$. Hence $\rho \subseteq \tau$. 
It follows from this that, if $\bar{\rho} \cap \mathscr{H}=\bar{\tau} \cap \mathscr{H}$, then $\rho=\tau$.

Remark. It follows from the proof of Theorem 2.4 that the map $\rho \mapsto \bar{\rho} \cap \mathscr{H}$ is an order isomorphism of the lattice of congruences on $T$, contained in $\mu^{*}$, onto the lattice of idempotent separating congruences on $S$. Eberhart and Williams [3] have shown that the map $\sigma \mapsto(\sigma \vee \mathscr{Y}) / \mathscr{Y}=\sigma^{*}$ is a lattice isomorphism in the other direction.

3. An application. A semigroup $S=S^{1}$ is called unit orthodox if it is orthodox and, for each $a \in S$, there is a unit $u$ such that $a=a u a$. It is uniquely unit orthodox if for each $a \in S$ there is a unique unit $u$ such that $a=a u a$.

RESULT 3.1.

(i) (Blyth \& McFadden [1]) Let Be be a band and let $G$ be a group acting on $B$, on the left by automorphisms. Then the semidirect product $B|\times| G$ of $B$ by $G$ is a uniquely unit orthodox semigroup: the multiplication is given by

$$
(a, g)(b, h)=(a+g b, h)
$$

Conversely, each uniquely unit orthodox semigroup has this form.

(ii) (McFadden [7]) Every unit orthodox semigroup is an idempotent separating homomorphic image of a uniquely unit orthodox semigroup.

In [7], McFadden gives a description for the idempotent separating congruences on the semidirect product of a band by a group. Thus, by Result 3.1(ii) he is able to characterize all unit orthodox semigroups. We shall apply the results of Section 2 and the known congruence theory for inverse semigroups to give an alternative, simpler description of idempotent separating congruences on uniquely unit orthodox semigroups.

Result 3.2 [7]. Let $B$ be $a$ band and let $G$ act on $B$ by automorphisms on the left. Then, in $B|\times| G$,

(i) $(a, g) \mathscr{H}(b, h) \Leftrightarrow a \mathscr{R} b$ and $\mathrm{g}^{-1} a \mathscr{L} h^{-1} b$;

(ii) $V(a, g)=g^{-1} D_{a} \times\left\{g^{-1}\right\}$ where $D_{a}$ denotes the $\mathscr{D}$-class of $B$ which contains a;

(iii) $(a, g) \mu(b, 1) \Leftrightarrow a=b+g b$ and $f=b+g f+b=b+g^{-1} f+b$ for all $f \leqslant b$. [Note that, from (i), $(a, \mathrm{~g}) \mathscr{H}(b, 1) \Leftrightarrow a=b+\mathrm{gb}$ and $\mathrm{g} D_{\mathrm{b}}=D_{\mathrm{b}}$.]

Since $G$ acts on $B$ by automorphisms, and Green's relations are defined by multiplication, $G$ induces an action on the semilattice $E$ of $\mathscr{D}$-classes of $B$. Thus we can form the semidirect product $E|\times| G$ of $E$ by $G$; this is an inverse semigroup. Indeed, from Result 3.2(ii), it is immediate that $E|\times| G$ is the maximum inverse homomorphic image of $B|\times| G$. Thus idempotent separating congruences on $B|\times| G$ are determined by idempotent separating congruences on $E|\times| G$. The next result, a special case of [6, Theorem 2.2], characterizes the latter.

RESULT 3.3 [6]. Let $E$ be a semilattice and $G$ be a group which acts on $E$ by automorphisms. Further let $\mathcal{N}=\left\{N_{\mathrm{A}}: A \in E\right\}$ be a family of subgroups of $G$ with the 
following properties:

(i) $A \leqslant C$ implies $N_{A} \supseteq N_{C}$;

(ii) $g N_{\mathrm{A}} g^{-1} \subseteq N_{\mathrm{gA}}$ for each $\mathrm{g} \in \mathrm{G}$;

(iii) $N_{A} \subseteq S_{B}=\{g \in G: g C=C$ for all $C \leqslant A\}$.

Then the relation $\rho_{\mathcal{N}}$ defined by

$$
(A, g) \rho_{\mathcal{N}}(C, h) \Leftrightarrow A=C \text { and } g h^{-1} \in N_{C}
$$

is an idempotent separating congruence on $E|\times| G$ and each such has this form for a unique family $\mathcal{N}$ of subgroups of $G$ which obeys (i), (ii) and (iii).

To apply Theorem 2.2 to obtain the idempotent separating congruences on a uniquely unit orthodox semigroup, it remains, as a consequence of Result 3.3, to describe the family $M$ of subgroups of $G$ corresponding to the congruence $\mu^{*}$ on $B|\times| G$.

Lemma 3.4. Let $B$ be $a$ band and let $G$ be a group which acts on $B$, on the left, by automorphisms. Let $E=B / \mathscr{D}$. Then

$(A, g) \mu^{*}(A, 1)$ if and only if

$$
g \in M_{A}=\left\{g \in G: f=b+g f+b=b+g^{-1} f+b \text { for all } f \leqslant b \text { and some } b \in A\right\} .
$$

Proof. By Lemma 2.1, $(A, g) \mu^{*}(A, 1)$ if and only if there exist $a, c \in A$ such that $(a, g)^{\prime} \mu(c, 1)^{\prime}$, for some inverses $(a, g)^{\prime},(c, 1)^{\prime}$ of $(a, g),(c, 1)$ respectively; that is, if and only if there exist $d, b \in A$ such that $\left(g^{-1} d, g^{-1}\right) \mu(b, 1)$. By Result 3.2 (iii), this occurs if and only if $g^{-1} d=b+g^{-1} b$ and $f=b+g^{-1} f+b=b+g f+b$ for all $f \leqslant b$. Hence $(A, g) \mu^{*}(A, 1)$ if and only if $g \in M_{A}$.

Note. If we define $M_{\mathrm{A}}(b)=\left\{g \in G: f=b+g f+b=b+g^{-1} f+b\right\}$, for $b \in A$ then it is straightforward to show that $M_{A}(b)=M_{A}(c)$ for all $b, c \in A$. Thus the qualifier "some $b \in A$ " in the definition of $M_{A}$ can be replaced by "for all $b \in A$ ".

If we now combine Lemma 3.4 and Results 3.2 and 3.3, and apply Theorem 2.4, we immediately obtain the following characterization of the idempotent separating congruences on $B|\times| G$.

THEOREM 3.5. Let $G$ be a group which acts on a band $B$ by automorphisms, on the left, and let $\mathcal{N}$ be a family of subgroups $\left\{N_{\mathrm{A}}: A \in E=B / \mathscr{D}\right\}$ with the following properties:

(i) $A \leqslant C$ implies $N_{A} \supseteq N_{C}$;

(ii) $g N_{A} g^{-1} \subseteq N_{\mathrm{gA}}$ for each $g \in G$;

(iii) $N_{\mathrm{A}} \subseteq M_{\mathrm{A}}=\left\{g \in G\right.$ : for all $b \in A, f=b+g f+b=b+g^{-1} f+b$ for all $\left.f \leqslant b\right\}$.

Then the relation $\rho_{\mathcal{N}}$ on $B|\times| G$ defined by

$$
(a, \mathrm{~g}) \rho_{\mathcal{N}}(b, h) \Leftrightarrow a \mathscr{R} b, \mathrm{~g}^{-1} a \mathscr{L} h^{-1} b \quad \text { and } g h^{-1} \in N_{\mathrm{A}} \text {, where } A \text { is the } \mathscr{D} \text {-class of } a \text {, }
$$

is an idempotent separating congruence on $B|\times| G$ and each such has this form for a unique family of subgroups of $G$ which obey (i), (ii) and (iii). 
D. B. McALISTER

\section{REFERENCES}

1. T. S. Blyth and R. B. McFadden, Unit orthodox semigroups, Glasgow Math. J. 24 (1983), 39-42.

2. A. H. Clifford and G. B. Preston, The algebraic theory of semigroups, Vols. 1 and 2 (American Mathematical Society, 1961 and 1967).

3. C. Eberhart and W. Williams, Congruences on an orthodox semigroup via the minimum inverse semigroup congruence, Glasgow Math. J. 18 (1977), 181-192.

4. T. E. Hall, On regular semigroups whose idempotents form a subsemigroup, Bull. Austral. Math. Soc. 1 (1969), 195-208.

5. J. M. Howie, Introduction to Semigroups (Academic Press, 1976).

6. D. B. McAlister, Groups, semilattices and inverse semigroups II, Trans. Amer. Math. Soc. 192 (1974), 351-370.

7. R. B. McFadden, Unit orthodox semigroups, to appear.

8. J. Meakin, Congruences on orthodox semigroups, J. Austral. Math. Soc. 12 (1971), 323-341.

9. W. D. Munn, A certain sublattice of the lattice of congruences on a regular semigroup, Proc. Cambridge Philos. Soc. 60 (1964), 385-391.

10. N. R. Reilly and H. E. Scheiblich, Congruences on regular semigroups, Pacific J. Math. 23 (1967), 349-360.

Department of Mathematical Sciences

NORTHERN ILLINOIS UNIVERSTTY

DEKALB

ILLINOIS 60115

U.S.A. 